\title{
Assessing Nursing Practices Regarding Standard Approaches on Post-Operative Wound Care Dressing
}

\author{
SAMIA SALEEM, MUHAMMAD HUSSAIN, MUHAMMAD AFZAL, SYED AMIR GILANI \\ Lahore School of Nursing, The University of Lahore - Pakistan \\ DOI 10.36552/pjns.v24i2.410
}

\begin{abstract}
Background and Objective: Post-operative delay wound dressing causes a wound contamination or delay wound healing process. Wound leakage and smell produce acute wounds changed into chronic wound conditions. This study assessed the nursing practices regarding standard approaches used for post-operative wound dressing and its care.

Materials and Methods: A cross-sectional observational study designed with study population $n=150$ in female nurses of two intensive care units and three surgical wards units: I-II-III. Data was collectedusing an adopted, observation checklist. Reliability tested by Cronbach alpha, 0.92. The mean scorewas categorized following rules, less than (1.5) was considered low (L), from (1.5-2.5) considered moderate $(M)$, and greater than (>2.5) was considered high $(H)$.

Results: The results indicated that $55.3 \%$ of nurses had 32 - 38 of age and $48.7 \%$ were BS nursing education. According to the mean score majority of nurses had medium $(M)$ and low $(L)$ wound dressing practices observed. Plaster not present (52\%) during dressing. $73.3 \%$ of nurses had not ensured privacy. The commonpractices were found, no handwashing before and after wound dressing.

Conclusion: The majority of the nurses were not following the standardized wound dressing checklist. According to the study, findings recommended the education sessions, seminars, frequent departmental observation, and psychomotor simulation practices that need to be discussed with clinical professionals.
\end{abstract}

Keywords: Wound Dressing, Dressing Material, Nursing Practices, Wound Infection.

\section{INTRODUCTION}

Most of the tertiary care hospitals found poor practices of wound care dressing and its management. Due to poor handling of post-surgical wound dressing materials. Due to these negligence's prolonged patient's hospital stays, delay wound healing process, and cost effect on their treatment of care. Postoperative surgical wounds not properly cleaned, and not utilized proper material of wound dressing. The poor nursing practices in wound management, prolong the wound healing process. This study was aimed to assess the nursing practices regarding standard approaches to post-operative wound care dressing. We assessed the association between Intensive Care Units and Surgical Wards the proper usage of available dressing material. Through the observation checklist, we assessed the nurses' practices regarding proper utilization of standard approaches for the postoperative wound care dressing. Study variables were measured through the checklist based on standard nursing practices. The study variables consisted of 4 parts, 1) Demographic, 2) Clinical Practices, 3) Dressing materials, and 4) Types of dressing material.

Every day, around 81,000 people acquires health care-associated infection in a hospital across Europe. Surgical site infection is the common type of HCAI (Health Care-Associated Infection), representing up to $20 \%$ of peoples were suffered from surgical site infection. Health care providers who provide the surgical wound dressing services required specialized knowledge, skills, and experience to ensure the evidence based wound care. ${ }^{1-8}$ 
Nurses play an important role in the prevention of surgical site infections and the healing of wounds through the dressing. Postoperative wound infection can arise from day one, but usually occurs between the fifth- and tenth-days following surgery, due to inadequate surgical wound care dressing ${ }^{9-15}$. Globally, the nursing profession requires a high degree of rational and standard-based psychomotor skills for competently and safely to perform various clinical procedures. The wound care is the fundamental skill of nursing care which taught in the nursing course. Most of the countries which were existed in wound care organizations. Including these organizations providing the standards of nursing wound care, such as Wound Ostomy and Continence Nurses Society (WOCN), American Professional Wound Care Association (APWCA), Australian Wound Management Association (AWMA). ${ }^{2}$ AWMA, evaluates the quality of patient care regarding wound care nursing practices. However, there is a need for an internationally accepted checklist used for wound dressing and nursing care practices. ${ }^{5}$

The aseptic technique contributes to evidencebased practice. Following on aseptic technique, to ensure the clear workplace, stainless steel trolley, sterile dressing pack, hand washing access, non-sterile gloves for old dressing removal, apron. For wound preparation, introduction, and maintain privacy if needed. Ensure patient comfort and position. ${ }^{14}$ After removing the old dressing, assessment of the wound condition, and record its condition for change the plan of care. Use sterile gloves after hand washing, and start from the dirty area and then move out to the clean air.Dressing material should use asper the prescription of the physician. ${ }^{16}$

Patient safety is an important aspect for every health care professional because they are all involved in the patient care. They provide healthcare services 24 hours a day to their client within an organization they worked. They account for $20 \%$ of all health-associated infections among surgical patients and $38 \%$ of Nosocomial Infection. A pilot study in Pakistan showed that $13 \%$ of patients undergoing elective surgery had surgical site infections due to unsatisfactory services provided by nurses of wound care $^{11}$. Quality care providingfor the post-operative wound dressing is a major concern for clinicians in all patient care environment. ${ }^{4}$

The evidence-based clinical practice recommended that acute wounds be treated routinely or at any change of dressing. Wounds must re-evaluate after receiving patients from the operating room or the previous shift. For wound care, must teach the patient and family. Wound treatment plans can include collaborative nursing care or a skilled nursing facility or other wound healing specialties like nutrition. ${ }^{6}$ In the United Kingdom; found that the wound dressing needs to change daily. Human resource is the most valuable asset of a health care system, lots of resources was used in the wound healing process. Surgical wound needs to asses frequently to make sure the wound is in the healing process ${ }^{9}$. During wound care services nurses need to consider the patient views, privacy and confidentiality. As acare provider to communicate the patient about the pros and cons of the wound dressing. The wound care clinician provides individualized nursing care and needs to involve the collaborative relationship between the client and professionals. Must need to guide how often the wounddressing will be changed. Educate the possible time of wound healing process. ${ }^{10}$

\section{MATERIALS AND METHODS}

\section{Study Design and Setting}

A cross-sectional observational study of postoperative wound dressing nurses' practices carried out from a Tertiary Care Hospital of Lahore, from February, 2020 to March 2020. The population was selected for study as a non-participant.

\section{Sample Size}

Non-probability sampling (Convenient). The Sample size was calculated $n=150$. It was calculated by Raosoft online, with a 5\% margin of error, $95 \%$ confidence level, population size $\mathrm{N}=250$, response distribution $50 \%$ ).

\section{Study Variables}

i) Independent Standard approaches on the postoperative wound dressing.

ii) Dependent Assess the Nursing Practices on the post-operative wound dressing.

\section{Ethical Consideration}

TheStudy was conducted after, Institutional approval from Lahore School of Nursing, The University of Lahore and, Organizational Ethical review committee from hospitals. Informed Consent was taken from the participants. Privacy and participant confidentiality 
were maintained throughout the study, only assess relevant components. Author also informed them to withdraw from the study at any time without any penalty.

\section{Inclusion Criteria}

The age limit was 18-35 years / Graduate, Diploma, Specialized nurses. Those nurses included who were willing to participate voluntarily with consents. Those nurses who were working in surgical units.

\section{Exclusion Criteria}

Following were excluded from the study: Head Nurses and, those nurses who were not working in Surgical ICU, Cardiac Surgery ICU, and post-surgical Ward. Nurses who were not willing to participate in this study were also excluded.

\section{Study Procedure and Data Collection}

Data collected $(n=30)$ through direct observation by the author at Surgical ICU, Surgical Wards (Units: I, II, III). Data was collected at Morning and Evening shift (Monday to Sunday). The duty roster was used to avoid the double observation of the study participants. Permission granted by sending emails. The tool items consisted of 4 parts, 1) Demographic Data, 2) Observational Characteristics $=32,3$ ) Types of dressing materials $=24$ items, 4 ) Dressing materials = 13 items. The standard nursing practices checklist of wound dressing reliability checked by Cronbach alpha and which was 0.92 .

\section{Data Analysis and Statistical Analysis}

Data was analyzed computing statistically through the Statistical Package of Social Sciences latest version 25, use of Excel Sheet. Include descriptive statistical analysis (Percentage and Frequency). Inferential statistical analysis (Chi-square test). However, overall results were scored according to the mean score. The results categorizedas follows: less than (1.5) was considered low (L), from (1.5 - 2.5) considered moderate $(\mathrm{M})$, and greater than (> 2.5) was considered $\operatorname{high}(\mathrm{H}){ }^{1}$

\section{RESULTS}

\section{Demographic Results}

This study participants were female nurses. In table 1 describes the demographic in which $32-38$ of age $(55.3 \%)$. The $16.7 \%$ of the participants were working in surgical ICU, $14.7 \%$ in Cardiac surgery ICU, 22\% in Surgical Ward U-I+HDU, 20.7\% Surgical Ward UI+HDU. The professional education levels showed that $49.3 \%$ of the nurse's education level of diploma, specialization, in BS. Degree $48.7 \%$. In job title, the $87.3 \%$ were registered nurses (see Figures 1, 2, 3, 4).

In figure 5, the results show that the frequencies of the practices responses. According to the mean scoring comparison the nursing wound care practices were observed low (L) $(2,10,11,12,13,14,17,18,19,20$, $23,25,26,31)$. Medium practices (M) were observed following the mean score comparison $(1,4,5,6,7,8$, $9,15,16,21,22,24,27,28,29,30,32)$.

\section{Assessment of Available Dressing Materials}

In figure (6) and table: 1, the results shows that assessment of availability the dressing materials: following the mean score levels. The Medium (M) (5, $8,9,10,11,13,14,18,19,20,22,23)$ and the low (L) observed available materials were $(1,2,3,4,6,7,12$, $15,16,17,21,24)$.

In table 1 above shows that the majority of dressing materials observed were used in the Intensive care unit during wound dressing rather than surgical wards. There was no association found between ICU and surgical wards because of the numbers of $(\mathrm{N})$ staff nurses not equal in both departments. There were no significant differences, almost, nurses in ICU and wards dust dressing trolley for carrying the equipment, put disposable gloves when opening wound, usage of toothed dissecting forceps for lift inner dressing off slowly, use normal saline, put on sterile gloves, clean the wound by using the forceps, use fresh gauze in one direction only. Both departments' nurses had good practices to use tape adhesive plaster to secure the dressing. There was no statistically significant difference in the usage of dressing materials between ICUs and Surgical Wards in wound dressing ( $p>$ $0.05)$. 

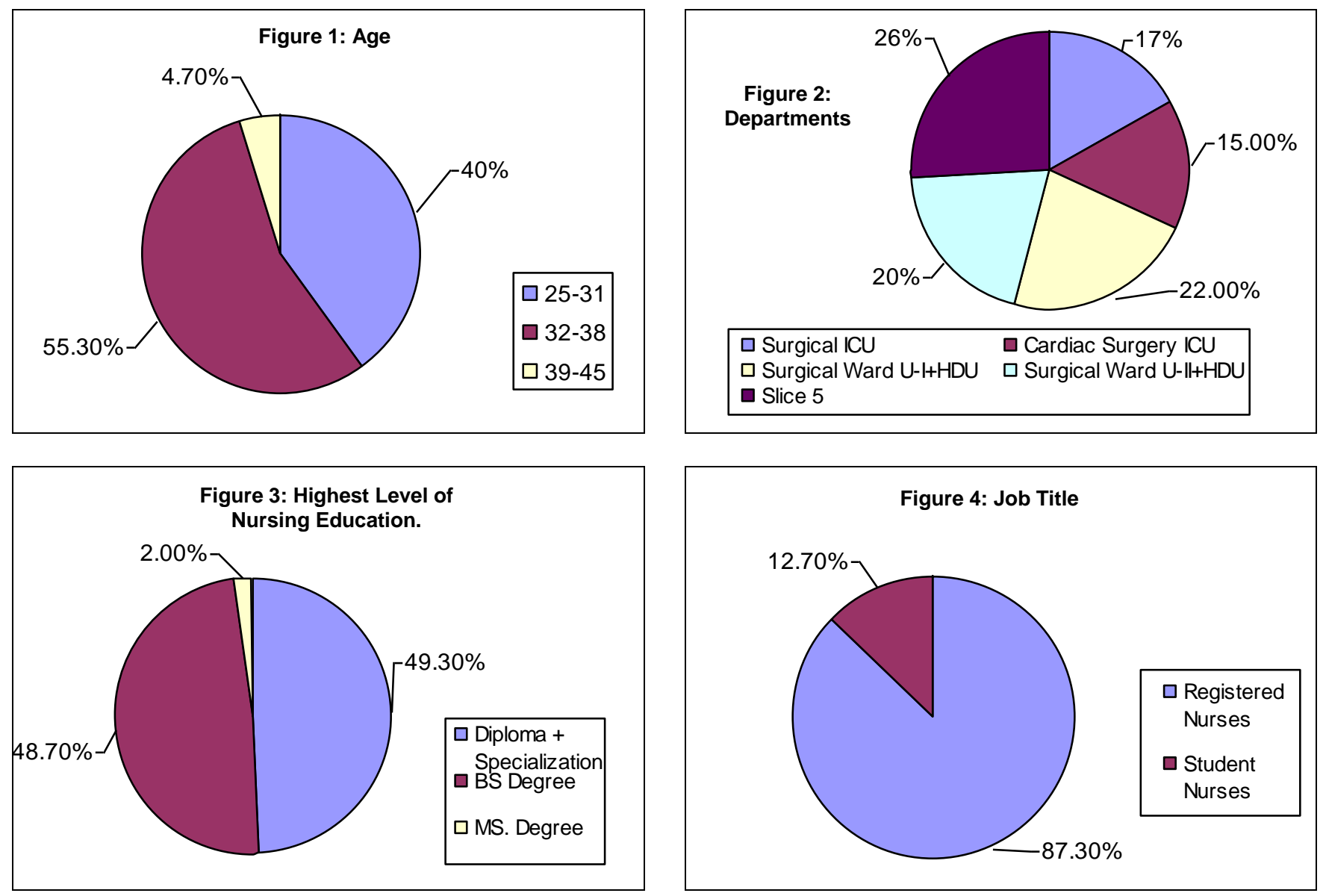

Figures 1-4: Percentages Representation of Age, Departments, Highest Level of Nursing Education and Job Title.

\section{Nursing Practices on Post-operative Wound Care}

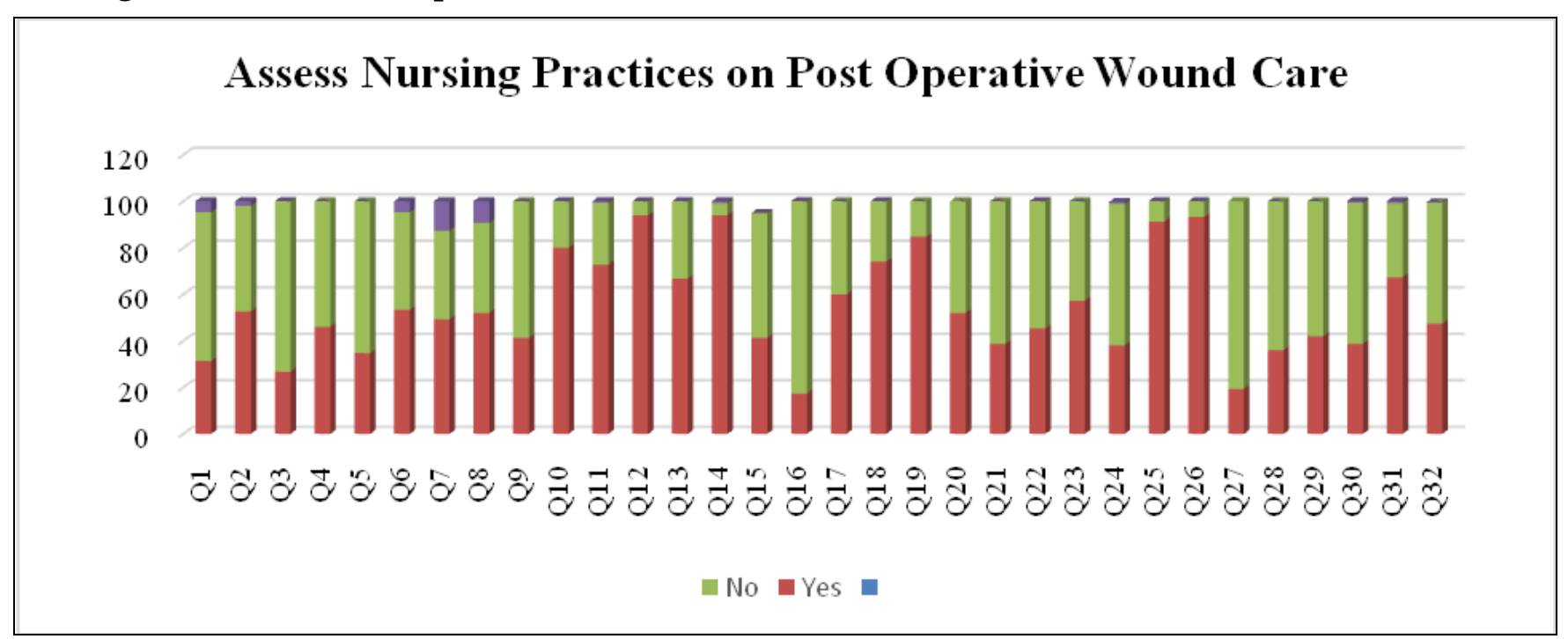

Fig. 5: Representation of the Frequencies of the Practices Responses. 


\section{Assessment of the Proper Use of the Available Dressing Materials}

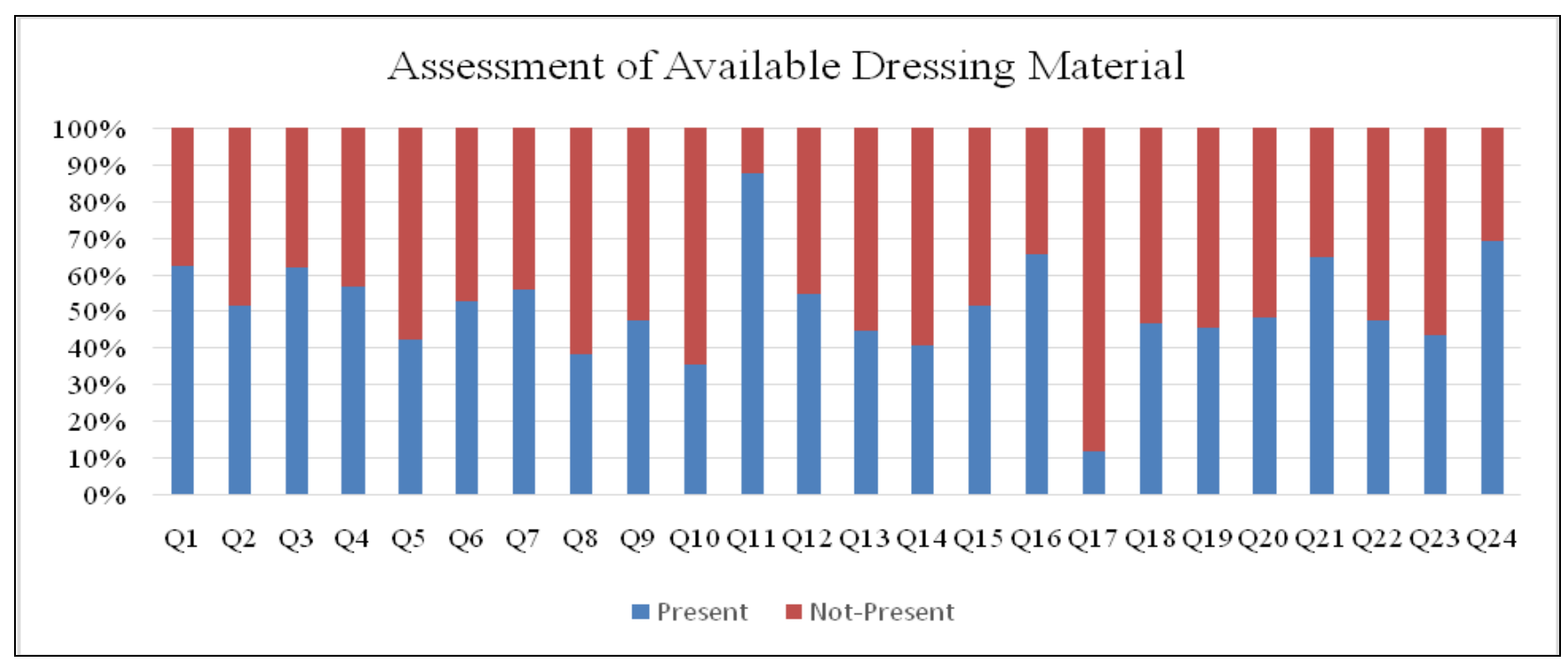

Fig. 6: Representation of the Assessment of Available Dressing Material.

Table 1: (Chi-square test to assess the association between ICUs and Wards nurse's proper usage of dressing material). $C$ $=$ Correct Performed $(N=46), I C=$ Incorrect Performed $(N=104)$.

\begin{tabular}{|c|l|l|l|l|}
\hline Sr\# & Dressing Materials & ICU (CP)-(IC) & Wards (CP)-(ICP) & $p$-Value \\
\hline 1. & Dust dressing Trolley for carrying equipment & $(29)-(17)$ & $(30)-(74)$ & 0.423 \\
\hline 2. & Place bag for soiled item within patient reach & $(24)-(22)$ & $(76)-(28)$ & 0.112 \\
\hline 3. & Put on disposable (clean) gloves when opening wound & $(34)-(12)$ & $(69)-(35)$ & 0.357 \\
\hline 4. & Using toothed dissecting forceps to lift inner dressing off slowly & $(35)-(11)$ & $(50)-(54)$ & 0.065 \\
\hline 5. & Use normal saline to sock the wound if dressing sticks on the wound & $(40)-(6)$ & $(39)-(65)$ & 0.061 \\
\hline 6. & Pour antiseptic solution into sterile receiver (kidney dish or gall pots) & $(42)-(4)$ & $(40)-(64)$ & 0.040 \\
\hline 7. & Put on sterile gloves while dressing the wound (Use one glove for one patient) & $(40)-(6)$ & $(32)-(72)$ & 0.080 \\
\hline 8. & Clean the wound by using forceps (non-toothed forceps) & $(25)-(21)$ & $(44)-(60)$ & 0.172 \\
\hline 9. & Use single sterile gauze in one direction only & $(30)-(16)$ & $(42)-(62)$ & 0.065 \\
\hline 10. & Use fresh gauze to dry the wound & $(37)-(9)$ & $(50)-(54)$ & 0.160 \\
\hline 11. & Apply dressing solution as prescribed & $(31)-(15)$ & $(55)-(49)$ & 0.022 \\
\hline 12. & Apply dry sterile dressing & $(37)-(9)$ & $(46)-(58)$ & 0.004 \\
\hline 13. & Use tape or adhesive plaster to secure dressing & $(44)-(2)$ & $(94)-(10)$ & 0.273 \\
\hline
\end{tabular}

\section{DISCUSSION}

A total of 150 nurses were included in this study. All nurses were female. The majority of the age group was $55.3 \%$ (32 - 38). Thirty-two percent of nurses were working during observed study variables, and sixtynine percent were surgical wards nurses. The majority of nursing education was $48.7 \%$ BS degree nurses and eighty-one percent of nurses were working as registered nurses. The nursing practices observed through a pre-tested standardized wound dressing checklist. In general, forty-three percent of participants were aged between 20-30 years, fifty-three percent had 
a degree in nursing and $17 \%$ had a master's degree in nursing. ${ }^{12}$

The majority of nurses were observed to ensure cleanliness and safety of the environment, placed waste bag for solid items within reach, seventy-four percent of nurses assist client/patient assumes a comfortable position during wound dressing. Discussion with a patient about procedure. Removing old dressing, use forceps to lifting the inner dressing off slowly, observe the nature of the wound. At national level variations are found in nursing practices. A study conducted in Lahore found a good level of nursing practices regarding prevention of surgical site infection $^{20}$ while another study conducted in Faisalabad showed low level of nurses' knowledge and practices. $^{21}$

Describing the procedure with the patient before the performing of the procedure is the ones of the patient's right. It gives the patient's sense of autonomy and encourages them to participate and understand their treatment of the issue as well as to manage in a better way. Patient and family education plays an important role in nursing practices. Many studies claimed that nurses tend to follow their hospital protocols. $^{3}$

In this study, the assessment of the available dressing materials, results were observed and reported according to mean score, which reveals the low presence of items during wound dressing. From the total count of 24 items, $60 \%$ mean score fall in Low (L) and 40\% meant of all in Medium (M). Most of the nurses were who had higher education; they rely on evidence-based practices rather than traditional practices. However, the studies recognized barriers the implementation of evidence-based practices, because of poor resource availability. ${ }^{16}$ At international level, a studyfound that $57.7 \%$ of nurses had poor practices about postop wound care. It was observed that they used of personal protective equipment's well, but dressing techniques were incorrect. ${ }^{17}$ Another two studies conducted in Ethiopia also foundinadequate nurses' practices in prevention of surgical site infection and dressing of acute wound. ${ }^{18,19}$

In our study results reveals that the majority of nurses were used aseptic sterile field during wound dressing and perform hand hygiene before and after dressing. Wear sterile gloves after removing the old dressing with disposable gloves. Use the antiseptic solution as prescribed for the wound dressing. Use sterile gauze and dry dressing the use of forceps. Sixty-four percent $(n=95)$ nurses had not to document the dressing record.

According to WHO guidelines hand hygiene should be performed before and after contact with the patient or any procedures to reduce the surgical site infection. Hand hygiene and aseptic measures are crucial to the application of standard precautions to prevent contamination. Documentation is an important communication method between all health care professionals to record and keep track of the wound healing process. Through the proper record of wound, assessment guided the multidisciplinary team to contribute to wound healing management and prevent infection. ${ }^{6}$

\section{SCOPE OF THE STUDY \& LIMITATIONS}

This study covered all aspects of observable nursing practices following the standards for post-operative wound dressing. This study was focused on nursing clinical practices. The study duration had a very short period of time. Every wound dressing was not to be observed. No, intervention and education implemented.

\section{CONCLUSION}

In this study, most of the nurses had a mean score of medium (M) significance level in wound dressing practices. Overall, the nurses had a lower mean score of wound dressing items which were not present like cotton swabs, dressing mackintoshes, dressing towels, mask and apron not present. No great association found between ICUs nurses, and surgical wards nursing wound dressing practices. To improve wound dressing, the nursing practices recommended utilizing standardized wound dressing protocols. Conduct the education sessions, seminars, and psychomotor enhancement simulation programs.

\section{ADDITIONAL INFORMATION ETHICAL REVIEW BOARD APPROVAL}

The letter was obtained from The Principal of Lahore School of Nursing, The University of Lahore. A Permission letter was received from the Nursing Superintendent of the study setting. Data were collected only for research purposes. This study was not harmful to any participants. Participation was completely voluntary. Data was kept confidential. 


\section{FUNDING}

No funding grant from the institute or another source.

\section{ACKNOWLEDGMENT}

I greatly appreciated the authors and participants for their immense contribution.

\section{REFERENCES}

1. Atiyah H, Khudhur K. Evaluation of nurses' practices toward postoperative wound dressing in surgical wards. Iraqi Nat J Spec. 2012; 1 (25): 29-39.

2. Ayello EA, Zulkowski K, Capezuti E, Jicman WH, Sibbald RG. Educating nurses in the United States about pressure injuries. Adv Skin Wound Care, 2017, 1; 30 (2): 83-94.

3. BaMohammed A, Mohidin S, George B, Al-Aidarous S. An Observational Study on Wound Dressing Performance among Nurses in Adult Units. J Nurs Health Sci. 2018; 3 (2): 01-06.

4. Beitz JM, Van Rijswijk L. A cross-sectional study to validate wound care algorithms for use by registered nurses. Ostomy Wound Manage. 2010; 1; 56 (4): 46.

5. Boga SM. Nursing Practices in the Prevention of PostOperative Wound Infection in Accordance with Evidence-Based Approach. Int J Caring Sci. 2019 May 1; 12 (2): 1228.

6. Desalew G, Geda B, Mengistie B, Demis A, Demis S. Surgical Site Infection Prevention Practices and Associated Factors among Nurses Working in Government Hospitals of Harari Regional State and Dire Dawa City Administration, Eastern Ethiopia. TMR Integr Nurs. 2019; 25; 3 (6): 214-25.

7. Ding S, Lin F, Marshall AP, Gillespie BM. Nurses' practice in preventing postoperative wound infections: an observational study. J Wound Care, 2017; 2; 26 (1): 28-37.

8. Harper. (2020). Collin dictionary. Com. Retrieved from [February 18, 2020]. https://www.collinsdictionary.com/dictionary/english

9. Hegarty J, Howson V, Wills $\mathrm{T}$, Creedon SA, McCluskey P, Lane A, Connolly A, Walshe N, Noonan B, Guidera F, Gallagher AG. Acute surgical wounddressing procedure: Description of the steps involved in the development and validation of an observational metric. Int Wound J. 2019; 16 (3): 641-8.

10. Lindholm C, Searle R. Wound management for the $21 \mathrm{st}$ century: combining effectiveness and efficiency. Int Wound J. 2016; 13: 5-15.

11. Lindsay E, Renyi R, Wilkie P, Valle F, White W, Maida V, Edwards H, Foster D. Patient-centred care: a call to action for wound management. J Wound Care, 2017; 2; 26 (11): 662-77.

12. Malik AZ. Surgical site infections after elective surgery in Pakistan: Surgipak Study. J Reg Med Campuses, 2015; 30; 19 (3): 209-14.

13. Mengesha A, Tewfik N, Argaw Z, Beletew B, Wudu M. Practice of and associated factors regarding prevention of surgical site infection among nurses working in the surgical units of public hospitals in Addis Ababa city, Ethiopia: A cross-sectional study. PloS One, 2020; 16; 15 (4): e0231270.

14. Mccluskey P, Mccarthy G. Nurses' Knowledge and Competence in Wound Management. Wounds UK. 2012; $1 ; 8$ (2).

15. Mwakanyamale AA, Mukaja AM, Ndomondo MD, Zenas JP, Stephen AM, Mika EZ. Nursing Practice on Post-Operative Wound Care in Surgical Wards at Muhimbili National Hospital, Dar-es-Salaam, Tanzania. J Nurs Open, 2019; 31; 9 (8): 870-90.

16. Pickering D, Marsden J. Techniques for aseptic dressing and procedures. Community Eye Health, 2015; 28 (89): 17.

17. Sadaf S, Inayat S, Afzal M, Hussain M. Nurse's knowledge and practice regarding prevention of surgical site infection at allied hospital Faisalabad. Int $\mathbf{J}$ Sci Eng Res. 2018; 9 (5): 351-69.

18. Sadia H, Kousar R, Azhar M, Waqas A, Gilani SA. Assessment of nurses' knowledge and practices regarding prevention of surgical site infection. Saudi J Med Med Sci. 2017; 3 (6): 585-95.

19. Sattar F, Sattar Z, Mohsin Zaman SA. Frequency of post-operative surgical site infections in a Tertiary care hospital in Abbottabad, Pakistan. J Cureus. 2019; 11 (3).

20. Ubbink DT, Brölmann FE, Go PM, Vermeulen H. Evidence-based care of acute wounds: a perspective. Adv Wound Care, 2015; 1; 4 (5): 286-94.

21. Welsh L. Wound care evidence, knowledge and education amongst nurses: a semi-systematic literature review. Int Wound J. 2018; 15 (1): 53-61. 


\section{Additional Information}

Disclosures: Authors report no conflict of interest.

Ethical Review Board Approval: The study was conformed to the ethical review board requirements.

Human Subjects: Consent was obtained by all patients/participants in this study.

Conflicts of Interest:

In compliance with the ICMJE uniform disclosure form, all authors declare the following:

Financial Relationships: All authors have declared that they have no financial relationships at present or within the previous three years with any organizations that might have an interest in the submitted work.

Other Relationships: All authors have declared that there are no other relationships or activities that could appear to have influenced the submitted work.

Address for Correspondence:

Samia Saleem

Lahore School of Nursing, The University of Lahore-Pakistan

Email: samiabhatti700@gmail.com

AUTHORSHIP AND CONTRIBUTION DECLARATION

\begin{tabular}{|l|l|l|}
\hline S\# & Author's Full Name and Affiliation & Intellectual Contribution to Paper in Term of: \\
\hline \multirow{4}{*}{1.} & \multirow{2}{*}{$\begin{array}{l}\text { Samia Saleem } \\
\text { Suhammad Afzal }\end{array}$} & Topic, Study design and methodology \\
\cline { 3 - 3 } & \multirow{2}{*}{ Syed Amir Gilani } & Paper Writing, referencing, data calculations \\
\cline { 3 - 3 } & & Data Collection and Data Calculations \\
\cline { 3 - 3 } & & Analysis of data and interpretation of results etc. \\
\cline { 3 - 3 } 2. & \multirow{2}{*}{ Muhammad Hussain } & Literature Review and Manuscript Writing \\
\hline
\end{tabular}

Date of Submission: 08-04-2020

Date of Revision: 15-05-2020

Date of Online Publishing: 30-06-2020

Date of Print: 30-07-2020 